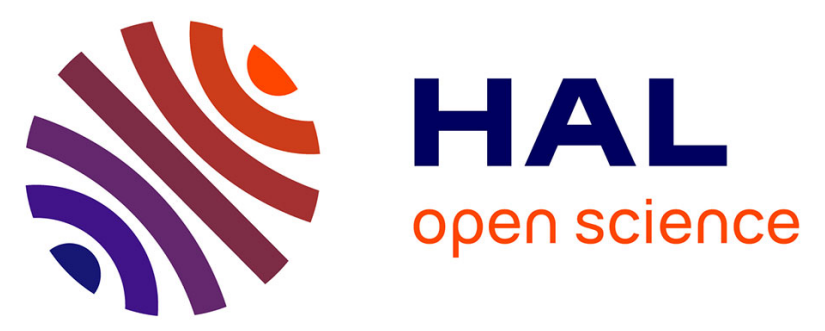

\title{
ACES/PHARAO: high performance space-to-ground and ground-to-ground clock comparison for fundamental physics
}

\author{
M. Lilley, E. Savalle, M. C Angonin, P. Delva, C. Guerlin, C. Le \\ Poncin-Lafitte, F. Meynadier, P. Wolf
}

\section{To cite this version:}

M. Lilley, E. Savalle, M. C Angonin, P. Delva, C. Guerlin, et al.. ACES/PHARAO: high performance space-to-ground and ground-to-ground clock comparison for fundamental physics. GPS Solutions, 2021, 25 (2), 10.1007/s10291-020-01058-y . hal-03185023

\section{HAL Id: hal-03185023 \\ https://hal.sorbonne-universite.fr/hal-03185023}

Submitted on 30 Mar 2021

HAL is a multi-disciplinary open access archive for the deposit and dissemination of scientific research documents, whether they are published or not. The documents may come from teaching and research institutions in France or abroad, or from public or private research centers.
L'archive ouverte pluridisciplinaire HAL, est destinée au dépôt et à la diffusion de documents scientifiques de niveau recherche, publiés ou non, émanant des établissements d'enseignement et de recherche français ou étrangers, des laboratoires publics ou privés. 


\title{
ACES/PHARAO: high performance space-to-ground and ground-to-ground clock comparison for fundamental physics
}

M. Lilley ${ }^{1 *}$, E. Savalle ${ }^{1}$, M.C. Angonin ${ }^{1}$, P. Delva ${ }^{1}$, C. Guerlin ${ }^{1,2}$, C. Le Poncin-Lafitte ${ }^{1}$, F. Meynadier ${ }^{3}$, P. Wolf ${ }^{1}$

${ }^{1}$ SYRTE, Observatoire de Paris, Université PSL, CNRS, Sorbonne Université, LNE, 61 avenue de l'Observatoire, 75014 Paris, France

${ }^{2}$ Laboratoire Kastler Brossel, ENS-Université PSL, CNRS, Sorbonne Université, Collège de France, 24 rue Lhomond, 75005 Paris, France

${ }^{3}$ Bureau International des Poids et Mesures, Pavillon de Breteuil, 92312 Sèvres Cedex, France

${ }^{*}$ Corresponding author: Marc Lilley, marc.lilley@obspm.fr

\begin{abstract}
The Atomic Clock Ensemble in Space (ACES) is a fundamental physics mission of the European Space Agency (ESA) to be launched in August 2021. It relies on a high-performance clock onboard the International Space Station (ISS), a network of high-performance clocks on ground, a dedicated two-way microwave link (MWL) enabling space-to-ground and groundto-ground clock comparisons, as well as an optical link (ELT). PHARAO/SHM (Projet d'Horloge Atomique par Refroidissement d'Atomes en Orbite/Space Hydrogen Maser), the clock onboard the ISS, has a relative frequency accuracy at the $10^{-16}$ level, a relative frequency stability (Allan deviation) equal to $10^{-13} / \sqrt{\tau}$ ( $\tau$ being the integration time in seconds) and a time deviation of 12 picoseconds after one day of integration. The MWL is designed to reach a time deviation below 7 ps after one day of integration. While space-to-ground clock comparisons will enable precise tests of the gravitational redshift, tests of deviations from General Relativity at the $10^{-6}$ level, and tests of local Lorentz invariance at the $10^{-10}$ level, ground-to-ground clock comparisons will enable a search of the time variation of fundamental constants with uncertainty at the $10^{-17}$ level after one year. In this contribution, we review the mission set up with a particular emphasis on the $M W L$, discuss the simulation and data analysis software developed to investigate mission performance, focusing on its primary scientific objective: the test of the gravitational redshift.
\end{abstract}




\section{Introduction}

The Atomic Clock Ensemble in Space (ACES) project is a Centre National d'Exploration Spatiale (CNES) and European Space Agency (ESA) led mission aiming to perform time comparisons between a high-performance clock aboard the International Space Station (ISS), the Projet d'Horloge Atomique par Refroidissement d'Atomes en Orbite/Space Hydrogen Maser, PHARAO/SHM, (Laurent et al. 2006), and high-performance clocks on the ground, in order to carry out tests of fundamental physics such as the gravitational redshift, Lorentz invariance and the possible variation of the fine structure constant. The launch is scheduled to occur in August 2021. In this review of the ACES experiment, we focus on the measurement of the gravitational redshift and possible deviations from General Relativity (GR) using ACES, and on a description of the microwave link data processing software used to perform space-toground time and frequency transfer. One particularity of this mission, which should be noted, is that the MWL is an integral part of the measurement process.

For ideal clocks, the fractional frequency difference between a clock on the ground and a clock in space is given by the difference in gravitational potential at the location of the clocks, and a second order Doppler effect,

$$
\Delta y(t)=\frac{d \tau^{g}}{d t}-\frac{d \tau^{s}}{d t}=\frac{\Delta v^{g}}{v^{g}}-\frac{\Delta v^{s}}{v^{s}}=\frac{1}{c^{2}}\left[U\left(t, \overrightarrow{x_{s}}\right)-U\left(t, \overrightarrow{x_{g}}\right)+\frac{v_{s}^{2}(t)}{2}-\frac{v_{g}^{2}(t)}{2}\right]+\mathrm{O}\left(c^{-4}\right)
$$

Here $\tau^{g}$ and $\tau^{S}$ are the proper times of the emitting clock on the ground, $g$, and the receiving clock in space, $s$, respectively and $t$ is coordinate time. The quantity $d \tau / d t$ is equal to the fractional frequency shift $\Delta v / v$. The quantity $U(t, \vec{x})$ is the Newtonian gravitational potential (with the convention that $U(t, \vec{x})>0$ ) and gives rise to the gravitational redshift, and $v^{2}(t) / 2 c^{2}$ is the second order Doppler effect, evaluated at the location of the ground clock and clock in space in a non-rotating coordinate system. The gravitational redshift for the ACES experiment is of order $\Delta U / c^{2} \sim 3.6 \times 10^{-11}$. Deviations from GR's gravitational redshift formula can be introduced and tested for against the ACES data by including a multiplicative factor $1+\alpha$ in the expression for $\Delta U / c^{2}$ so that it becomes $(1+\alpha) \Delta U / c^{2}$. If the value of $\alpha$ is found to significantly different from zero when confronting the data with the theoretical model, a deviation from GR is detected.

The time scale of high stability and accuracy onboard the ISS uses a unique cold-atom space clock (PHARAO) developed by CNES in collaboration with the Laboratoire National de 
Métrologie et d'Essais-Système de Référence Temps-Espace (LNE-SYRTE) and an SHM. The time and frequency comparisons with an international network of ground clocks will be made possible by a MWL and optical links (the European Laser Timing, ELT). The level of precision with which tests of fundamental physics will be performed will be limited by the performance of the clocks and by the MWL and ELT links. The Allan deviation of the clock ensemble will be of the order $10^{-13} / \sqrt{\tau}$, where $\tau$ is integration time, which corresponds to $3 \times 10^{-16}$ after one day of integration and the time deviation will be of order $4 \times 10^{-14} \sqrt{\tau}$, corresponding to 12 ps after one day of integration. The fractional frequency accuracy will be of the order $10^{-16}$. The ACES MWL is designed to exceed the long-term stability of PHARAO/SHM by a factor 3. The expected gravitational frequency shift between ground segment (GS) clocks and PHARAO/SHM for an ISS orbit of altitude $400 \mathrm{~km}$ is of order $3.6 \times 10^{-11}$. The performance of the ACES experiment should, therefore, allow a test of the gravitational redshift at the level of a few parts in $10^{6}$. The flight segment of the MWL is currently being tested on ground in end-to-end configurations with the MWL ground terminals while tests of the clock ensemble PHARAO/SHM on ground give an Allan deviation of order $3 \times 10^{-13} / \sqrt{\tau}$ (Cacciapuoti et al., 2020).

\section{PHARAO and SHM}

The ACES mission is a unique opportunity to take advantage of advances in atomic clock performance made in the past two decades in order to perform worldwide clock comparisons and allow improved tests of fundamental laws such as GR. In ACES, the clock ensemble aboard the ISS is the combination of PHARAO, and SHM (Laurent et al. 2006). PHARAO is a laser-cooled Cesium clock. The main sources of frequency shifts of PHARAO are the collisions between cold atoms and the first-order Doppler effect. A short-term servo loop steers PHARAO's local oscillator towards the clock signal of SHM with a typical time constant of a few seconds while a long-term servo loop corrects SHM's 100 MHz clock signal with a time constant of a few hundred seconds. The ACES clock signal is the combination of both the PHARAO and SHM signals. It has the medium-term stability of the SHM and the long-term stability of PHARAO. The Allan deviation is therefore limited by the performance of SHM on medium time scales and by the performance of PHARAO on long time scales for 
an overall relative frequency stability equal to roughly $10^{-13} / \sqrt{\tau}$ and a time deviation of 12 ps after one day of integration, see Fig. 1. As mentioned in the introduction, the performance of the PHARAO/SHM clock ensemble sets the requirement on the performance of the MWL, as depicted by the green curve in the lower plot of the figure while the time deviation of the ELT network is depicted in blue.
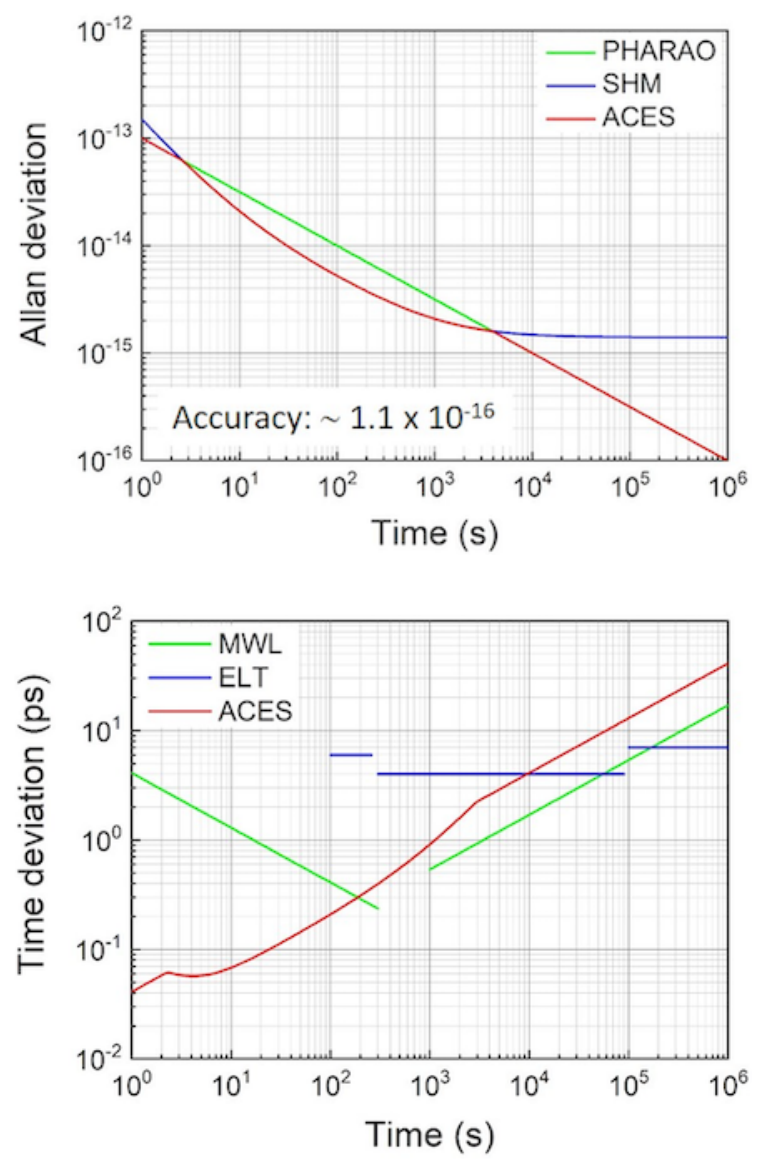

Fig. 1 Allan deviation (top) for PHARAO, SHM and the clock ensemble aboard the ISS, and the corresponding time deviation (bottom) for the ACES clock ensemble and the requirements on the MWL and the ELT (Cacciapuoti et al. 2020).

\section{Microwave Link}

The MWL provides the means to compare PHARAO/SHM to ground clocks. It is composed of signals at three frequencies: an uplink from ground clocks to the ISS at $13.5 \mathrm{GHz}$, and two downlinks from the ISS to ground clocks at $14.7 \mathrm{GHz}$ and $2.2 \mathrm{GHz}$. For the uplink and downlink signals, 1.0 pulse-per-second (PPS), 12.5 PPS, PRN (Pseudo Random Noise) code, and carrier 
phase observables at frequencies listed in Table 1 are available and enable the calculation of the pseudo-time-of-flight (pToF) for each link between the ground and PHARAO with picosecond accuracy at 80 ms intervals, set by the local 12.5 PPS signal. This pToF contains information about both the time-of-flight of the signal and the difference between the times given by the emitter and receiver clocks. From the pToF, one is able to determine the desynchronization between the ground and space clocks using a model of the space-time geometry, a model for the propagation of the signal through the earth's atmosphere, orbitography, and by taking into account instrumental delays provided by on-ground calibration.

Table 1 Carrier, code and resulting beat note frequencies for the uplink and downlink signals. The superscript " $A$ " can take values "ca" (carrier) or "co" (code).

\begin{tabular}{|c|c|c|c|c|}
\hline & $\begin{array}{c}\text { Carrier } \\
\text { Uplink } \\
(\mathrm{MHz})\end{array}$ & $\begin{array}{c}\text { Carrier } \\
\text { Downlink 1 } \\
(\mathrm{MHz})\end{array}$ & $\begin{array}{c}\text { Carrier } \\
\text { Downlink } \\
2(\mathrm{MHz})\end{array}$ & Code \\
& 13475.000 & 14703.333 & 2240.000 & 100.000 \\
\hline$f^{A}$ & 13474.270 & 14702.604 & 2239.270 & 100.195 \\
\hline$f_{\text {local }}$ & 0.729 & 0.729 & 0.729 & 0.195 \\
\hline$f_{b}^{A}= \pm\left(f_{\text {local }}-f^{A}\right)$ & & & & \\
\hline
\end{tabular}

Two-Way Link and the $\Lambda$ Configuration

This subsection describes how desynchronization can be obtained from the pToF's of the uplink and the two downlinks with high precision in a specific configuration called the $\Lambda$ configuration. Fig. $\mathbf{2}$ is a schematic representation of the MWL measurement. A one-way uplink is represented in red while the two one-way downlinks are represented in dark and light blue. The two-way link is obtained by combining data from all three links, and the $\Lambda$ configuration is obtained when time $t_{2}=t_{3}=t_{5}$. As we explain below, the presence of the second downlink enables the determination of ionospheric delays. 


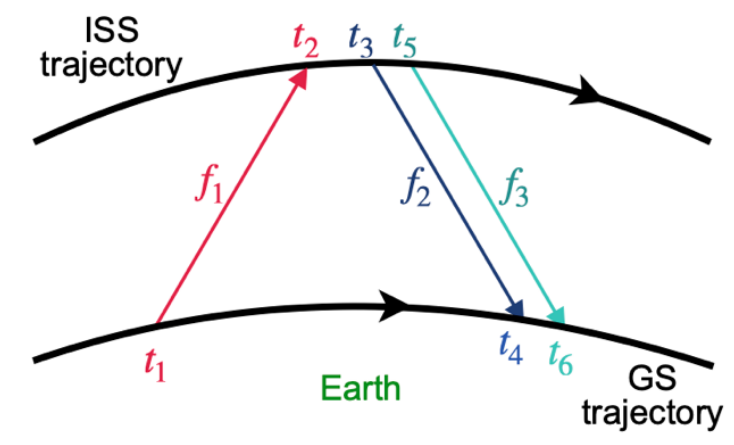

Fig. 2 One-way uplink represented in red from $t_{1}$ to $t_{2}$, and two one-way downlinks represented in dark blue from $t_{3}$ to $t_{4}$ and in light blue from $t_{5}$ to $t_{6}$. The $\Lambda$ configuration, obtained by setting $t_{2}=t_{3}=t_{5}$, removes, to first order, the range, tropospheric delay, and Shapiro effect.

In the one-way uplink, the fundamental quantity is the pToF, given by:

$\Delta \tau^{s}\left(\tau^{s}\left(t_{2}\right)\right)=\tau^{g}\left(t_{1}\right)-\tau^{s}\left(t_{2}\right)$

where $\tau^{g}$ and $\tau^{S}$ are the proper times of the emitting clock on the ground, $g$, and the receiving clock in space, $s$, respectively and where $t$ is coordinate time, with $t_{1}$ and $t_{2}$ the coordinate times of emission and reception respectively. A similar expression for the pToF of the downlink signal $f_{2}$ reads

$\Delta \tau^{g}\left(\tau^{g}\left(t_{4}\right)\right)=\tau^{s}\left(t_{3}\right)-\tau^{g}\left(t_{4}\right)$

For a single link, the desynchronization between clocks $g$ and $s$ is given by:

$\delta\left(t_{2}\right)=\tau^{s}\left(t_{2}\right)-\tau^{g}\left(t_{2}\right)=-\Delta \tau^{s}\left(\tau^{s}\left(t_{2}\right)\right)-\left[T_{12}+\left[\Delta_{1}^{g}+\Delta_{1}^{s}\right]^{t}\right]^{g}$

In this expression, $T_{12}=t_{2}-t_{1}$ is the time-of-flight between $g$ and $s$ in coordinate time, $\Delta_{1}^{g}$ and $\Delta_{1}^{S}$ are internal delays introduced in the uplink by the hardware, while [·] ${ }^{g / s}$ denotes a coordinate to proper time transformation, and $[\cdot]^{t}$ a proper to coordinate time transformation.

The time-of-flight $T_{i j}=t_{j}-t_{i}$ is modeled by the sum of four terms:

$T_{i j}=\frac{R_{i j}}{c}+\Delta_{i j}^{i o n o}(f)+\Delta_{i j}^{\text {tropo }}+\Delta_{i j}^{\text {Shapiro }}$

where $R_{i j}$ is the geometric range and where the three additional terms are the delays induced by the ionosphere, the troposphere and the effect of the Earth's gravitational field on light 
propagation, the Shapiro effect (see Duchayne et al. 2009). Some orders of magnitude for the individual contributions to $T_{i j}$ for frequencies $f_{1}$ and $f_{2}$ are $R_{i j} / c \sim 10^{-3} \mathrm{~s}, \Delta_{i j}^{i o n o} \sim 10^{-9} \mathrm{~s}$, $\Delta_{i j}^{\text {tropo }} \sim 10^{-7} \mathrm{~s}$, and $\Delta_{i j}^{\text {Shapiro }} \sim 10^{-11} \mathrm{~s}$. For the downward link, from $s$ to $g$, desynchronization is given by:

$\delta\left(t_{3}\right)=\tau^{s}\left(t_{3}\right)-\tau^{g}\left(t_{3}\right)=\Delta \tau^{g}\left(\tau^{g}\left(t_{4}\right)\right)+\left[T_{34}+\left[\Delta_{2}^{g}+\Delta_{2}^{s}\right]^{t}\right]^{g}$

where $t_{3}$ and $t_{4}$ are the coordinate times of emission and reception respectively, and where $\Delta_{2}^{g}$ and $\Delta_{2}^{s}$ are downlink internal delays for $f_{2}$. The $\Lambda$ configuration is defined by $t_{2}=t_{3}$, and $T_{23}=0$ (this latter equality being only approximately verified in practice). In this case, one obtains

$\delta\left(t_{2}\right)=\frac{1}{2}\left(\Delta \tau^{g}\left(\tau^{g}\left(t_{4}\right)\right)+\Delta_{2}^{g}+\Delta_{2}^{S}-\Delta \tau^{S}\left(\tau^{s}\left(t_{2}\right)\right)-\Delta_{1}^{g}-\Delta_{1}^{S}+\left[T_{34}-T_{12}\right]^{g}\right)$

As can be seen in this expression, in the two-way link for desynchronization in the $\Lambda$ configuration, the difference of the times-of-flight $T_{34}-T_{12}$ appears. By canceling leading order terms, this difference allows one to minimize the impact of errors in the determination of the time-of-flight, the Shapiro effect and the tropospheric delay (Duchayne et al. 2009). Note also that in the expression for $T_{i j}, \Delta_{i j}^{i o n o}(f)$ is frequency-dependent. Given that, by design, the uplink and downlink operate at different frequencies, $f_{1}$ and $f_{2}$, the $\Lambda$ configuration does not cancel the leading order term in the delay introduced by the ionosphere. As we shall see in a forthcoming section, it is the existence of the additional downlink signal at frequency $f_{3}$ that allows the precise determination of $\Delta_{i j}^{i o n o}(f)$. In the following subsection, we discuss the computation of the terms on the right-hand side of the expression for desynchronization.

Desynchronization

In order to synthesize the $\Lambda$ configuration from the non- $\Lambda$ configuration that is realized in practice, a first approximation of range and desynchronization in the non- $\Lambda$ configuration is required. This computation requires the determination of the pToF for the uplink and the two downlinks from the raw data. The highest precision is obtained by using the carrier signal pToF, which can be written as (Meynadier et al. 2018): 
$\Delta \tau^{c a}\left(\tau^{c a}\right)=-\frac{f_{b}^{c a}}{f^{c a}} \tau^{c a}+\frac{1}{f^{c a}} \frac{\phi_{b}^{c a}\left(\tau^{c a}\right)}{2 \pi}+\frac{k}{f^{c a}}+\frac{1}{f^{c a}} \frac{\phi_{0}^{c a}}{2 \pi}$

In this expression, $\tau^{c a}$ is the proper time of the carrier signal at reception, $\phi_{b}^{c a}$ is the phase of the beat note formed from the emitted carrier signal and the local clock, at frequency $f_{b}^{c a}=0.729 \mathrm{MHz}$ (see Table 1) while $k$ and $\phi_{0}^{c a}$ are the integer and fractional part of the carrier phase ambiguity, respectively. The signal is sampled with a resolution limited by the frequency of the receiver clock, $f_{\text {clock }}=100.195 \mathrm{MHz}$. This sets the precision of the proper time $\tau$ to $10 \mathrm{~ns}$, such that both the precision of $\left(f_{b}^{c a} / f^{c a}\right) \tau^{c a}$ and that of $\left(\phi_{b}^{c a}(\tau) / 2 \pi\right) f^{c a}$ are of order $0.5 \mathrm{ps}$. The integer $k$ can be computed unambiguously from the code data as long as two conditions are fulfilled. First, the code's pToF $\Delta \tau^{c o}\left(\tau^{c o}\right)$ can be interpolated at times $\tau^{c a}$ to better than a cycle of the carrier, i.e. about $70 \mathrm{ps}$. Second, $\Delta \tau^{c o}\left(\tau^{c a}\right)$ should be determined with a precision better than 70 ps, such that $f_{c a} \delta\left(\Delta \tau^{c o}\left(\tau^{c a}\right)\right) \ll 1$. Taking some safety margin, we set this requirement to $20 \mathrm{ps}$. The precision of the fractional part of the carrier phase ambiguity, $\phi_{0}^{c a}$, is also limited to $20 \mathrm{ps}$, a factor 40 greater than the $0.5 \mathrm{ps}$ requirement. This being said, $\phi_{0}^{c a}$ is common to all passes of the ISS, as long as the MWL remains turned on. For most scientific objectives of the mission, which rely on the differences of pToF measurements, this initial phase cancels out. For those scientific observables that rely on the absolute values of the pToF measurements, the overall uncertainty will be limited by the absolute uncertainty on $\Delta \tau^{c o}$ after averaging over all measurements and taking into account uncertainties from the calibration of internal hardware delays.

As explained in the previous paragraph, the code's pToF is required to have a $20 \mathrm{ps}$ or better in order to resolve the carrier phase ambiguity. There are two ways to compute the code's pToF from the MWL raw data. The first method is straightforward and relies on the existence of a frame counter at frequency $f_{f c}=2,500 \mathrm{~Hz}$, resetting to zero once per second, and a chip counter at frequency $f_{c c}=10^{8} \mathrm{~Hz}$, resetting to zero once it reaches 40,000 such that the time of emission of the code is known unambiguously. The time of arrival of the code is measured using the zero crossing of the beat note formed from the difference of the incoming signal (divided at $100 \mathrm{MHz}$ ) and the local clock at $100.195 \mathrm{MHz}$ (see Table 1). This procedure introduces an absolute error that lies between 0 and $\left(1-f_{c c} / f_{\text {local }}\right) / f_{\text {local }} \sim 20$ ps, for a single measurement ( $80 \mathrm{~ms}$ sampling), which averages down during the ISS pass. The second method is much more involved and does not make use of the frame and chip counters, but instead relies on a recurrence relation between successive pToFs that are derived by 
exploiting the phase variation equality of the emitted and received signal, and uses the 1PPS data to resolve the code phase ambiguity (Delva et al. 2015).

Once $\Delta \tau^{c a}\left(\tau^{c a}\right)$ is known with precision at the $0.5 \mathrm{ps}$ level for all three links, the quantity $\left[T_{34}-T_{12}\right]^{g}$ can be computed (Duchayne et al. 2009; Meynadier et al. 2018). The transformation from coordinate time to proper time, $[\cdot]^{g}$, is given by:

$$
[\cdot]^{g}=1-\frac{G M}{r_{g}(t) c^{2}}-\frac{v_{g}^{2}(t)}{2 c^{2}}
$$

In this expression, $G$ is the gravitational constant, $M$ is the mass of the earth, $r_{g}(t)$ and $v_{g}(t)$ are the ICRS radial coordinate and coordinate velocity of the ground clock at coordinate time $t$, respectively. The quantities $\left(G M / r_{g} c^{2}\right) T_{34}$ and $\left(G M / r_{g} c^{2}\right) T_{12}$ are of order $0.6 \mathrm{ps}$ while the terms $\left(v_{g}^{2} / 2 c^{2}\right) T_{12}$ and $\left(v_{g}^{2} / 2 c^{2}\right) T_{34}$ are of order 0.002 ps. Therefore, while the velocity term can be neglected, the amplitude of the gravitational term is comparable to the precision required and has to be accounted for. Next, we discuss the calculation of the time intervals $T_{12}$ and $T_{34}$. As already mentioned, the expression for $T_{i j}$ is the sum of four terms: the geometric range, the Shapiro effect, the tropospheric and ionospheric delays. While the geometric range and Shapiro delays are computed from orbit data, the ionospheric and tropospheric delays are determined using a Chapman layer STEC model and a Saastamoinen model respectively (Duchayne et al. 2009; Meynadier et al. 2018).

In order to calculate the ionospheric delay for all three links, we first solve for the Slant Total Electron Content (STEC), $S$, from the two downlinks, using, on the one hand (Bassiri \& Hajj, 1993):

$\Delta_{i j}^{i o n o}(f) \cong \frac{\alpha}{c f^{2}} S\left(1+\frac{\beta c}{f} B_{0} \cos \theta_{0}\right)$

where the coefficients $\alpha$ and $\beta$ are numerical coefficients that depend on whether the formula applies to code or carrier, where $B_{0}$ and $\theta_{0}$ are the earth's magnetic field and the angle subtended by $\vec{B}$ and the line-of-sight, and on the other hand, the difference of the pToF's at frequencies $f_{2}$ and $f_{3}$, which can be approximated by:

$\Delta_{56}^{i o n o}\left(f_{3}\right)-\Delta_{34}^{i o n o}\left(f_{2}\right)=\Delta \tau^{g}\left(\tau^{g}\left(t_{4}\right)\right)-\Delta \tau^{g}\left(\tau^{g}\left(t_{6}\right)\right)+\frac{R_{34}-R_{56}}{c}$

As can be seen from this expression, although downlink geometric ranges for $f_{2}$ and $f_{3}$ (and thus orbital data) is necessary to compute the value of $S$, and ionospheric delays are required 
to compute geometric range, it is the difference of the geometric range measurements for the two downlinks, divided by the speed of light that appears on the right-hand-side of the equation for $\Delta_{56}^{i o n o}\left(f_{3}\right)-\Delta_{34}^{i o n o}\left(f_{2}\right)$. This is a sub-leading correction, so that $S$ is mostly determined from the difference of $\Delta \tau^{g}\left(\tau^{g}\left(t_{4}\right)\right)-\Delta \tau^{g}\left(\tau^{g}\left(t_{6}\right)\right)$, and is then used to correct for both the uplink and downlink ionospheric delays.

At leading order, and as already mentioned, the difference $\Delta_{34}^{\text {tropo }}-\Delta_{12}^{\text {tropo }}$ cancels out in the expression for desynchronization, up to small corrections caused by tropospheric dispersive effects (Hobiger et al. 2013) which we neglect in this work, and up to small corrections proportional to $v_{S} / c$ which are due to the difference in the path between the uplink and the downlink. This being said, estimates of the uplink and downlink tropospheric delays are needed to compute the ToF information required to form the $\Lambda$ configuration. The two-way tropospheric delay appears by summing the observables at frequencies $f_{1}$ and $f_{2}$, and by using the expression for $T_{i j}$. It reads:

$$
\begin{aligned}
\Delta_{12}^{\text {tropo }}+\Delta_{34}^{\text {tropo }}= & -\frac{R_{12}+R_{34}}{c}-\left(1+\frac{G M}{r_{g}\left(t_{2}\right) c^{2}}\right)\left(\Delta \tau^{S}\left(t_{2}\right)+\Delta \tau^{g}\left(t_{4}\right)\right)-\left(\Delta_{12}^{\text {iono }}\left(f_{1}\right)+\right. \\
& \left.\Delta_{34}^{\text {iono }}\left(f_{2}\right)\right)
\end{aligned}
$$

As can be seen from the right-hand side of this expression, and by contrast with the equation for the STEC, the sum of geometric ranges for the uplink and downlink at $f_{1}$ and $f_{2}$ is required. The zeroth-order errors on the quantities $R_{i j}$ computed from orbitography data appear in a sum rather than in a difference and no longer cancel, as was the case in the expression for desynchronization or for the STEC calculation.

In order to break the link between tropospheric delay and geometric range, we obtain tropospheric delay using a Saastamoinen model (Saastamoinen 1973) and external atmospheric data:

$\Delta^{\text {tropo }}=\frac{2.277 \times 10^{-3}}{c \cos z}\left[p+\left(0.05+\frac{1255}{T}\right) e-(\tan z)^{2}\right]$

where $p$ is the atmospheric pressure, $T$ is the atmospheric temperature, $e$ is the water vapor partial pressure and $z$ is the angle between the line-of-sight and zenith and is calculated from the positions of the ISS and the ground station in the International Celestial Reference Frame (ICRF). 
Once a first determination of range and desynchronization in the non- $\Lambda$ configuration is obtained, the $\Lambda$ configuration is obtained by interpolation of the pToF's. If one chooses to interpolate the $f_{1}$ pToFs, then the $f_{1}$ data is interpolated at times:

$\tau_{2}^{\text {int }}=\tau_{4}-\delta\left(\tau_{4}\right)-\left(R_{34}\left(\tau_{4}\right)+\Delta_{34}^{\text {tropo }}+\Delta_{34}^{\text {iono }}\right)$

In other words, the proper time of reception $\tau_{2}^{i n t}$ for the uplink data in the $\Lambda$ configuration is equal to the proper time of reception $\tau_{4}$ for the $f_{2}$ downlink data minus the ToF between the ISS and the ground terminal at $\tau_{4}$ minus the clock desynchronization.

\section{Timing and Frequency Errors}

On the one hand, and as can be seen in the expression for desynchronization, the timing error is related to the determination of the quantity $T_{12}-T_{34}$. At leading order, this difference is proportional to the range $R$ and to the quantity $T_{23}$, which is related to the calibration uncertainty on the internal delays. The timing error, therefore, depends on the uncertainty in $T_{23}, \delta\left(T_{23}\right)$, and on the uncertainty in the position of the ISS, $\delta(R)$ (Duchayne et al. 2009). By modeling both $\delta\left(T_{23}\right)$ and $\delta(R)$, computing the temporal Allan deviation and comparing it with requirements, Duchayne et al. (2009) have established that $T_{23} \leq 10^{-6}$ s and $\delta\left(T_{23}\right) \leq$ $0.1 \mu$ s while Meynadier et a. (2018) find a constraint on the precision of the orbits of order 1 $\mathrm{km}$.

On the other hand, the gravitational redshift causes a difference in the frequencies of the GS and ISS clocks, which corresponds to a variation of desynchronization. While timing errors related to $T_{12}-T_{34}$ drop out of the corresponding expression, errors in the positioning and velocity of the ISS will induce errors on the modeling of the orbiting clock's relativistic frequency corrections along its trajectory. Constraints on $\delta(R)$ are then obtained by comparing the modified Allan deviation of the measurements involving such ISS positioning errors with requirements. One finds $\delta(R) \leq 10 \mathrm{~m}$ (Duchayne et al. 2009).

To summarize, a reliable determination of ISS orbits and a reliable calibration of internal delays are required both to compute the corrections to desynchronization in the data processing step and to compute the second order Doppler effect and gravitational redshift in the data analysis step. 
In Table 2, we gather our current understanding of the ACES error budget by giving the Allan deviations or time deviations associated with the main sources of error in the experiment: PHARAO/SHM, the MWL, the impact of orbitography on desynchronization (Meynadier et al., 2018), and the impact of orbitography on the redshift error (Savalle et al., 2018). As mentioned above, an additional constraint on the calibration of internal delays imposes $T_{23} \leq 10^{-6} \mathrm{~s}$ and $\delta\left(T_{23}\right) \leq 0.1 \mu \mathrm{s}$; see Duchayne et al. (2009). For long integration times, the error is dominated by PHARAO/SHM while for short integration times, it is dominated by the MWL. The impact of orbitography errors on the MWL is relevant only on short time scales corresponding to the duration of a pass. The impact of orbital errors on the MWL's time deviation remains below MWL requirements for errors smaller than $1 \mathrm{~km}$. The impact of orbital errors on the redshift error, independently from errors made on the determination of desynchronization, remain below PHARAO/SHM's Allan deviation for errors smaller than $100 \mathrm{~m}$.

Table 2: Allan deviations or temporal deviations for the main sources of uncertainty in the gravitational redshift test performed witht the ACES experiment.

\begin{tabular}{|c|c|c|}
\hline & Time deviation & Allan deviation \\
\hline PHARAO/SHM stability & & $\begin{array}{c}10^{-13} / \sqrt{\tau} \\
\text { All } \tau\end{array}$ \\
\hline MWL stability requirement & $\begin{array}{l}7 \times 10^{-12} / \sqrt{\tau} \\
\text { for } \tau<10^{3} \mathrm{sec}\end{array}$ & \\
\hline $\begin{array}{l}\text { Impact of orbitography } \\
\text { on redshift error } \\
\text { Savalle et al. (2019) }\end{array}$ & & $\begin{array}{c}9 \times 10^{-12} / \tau \\
\text { for } 100 \mathrm{~m} \text { orbital error } \\
\text { and } \tau \geq 10^{3} \mathrm{~s}\end{array}$ \\
\hline $\begin{array}{l}\text { Impact of orbitography } \\
\text { on MWL stability } \\
\text { Meynadier et al. (2018) }\end{array}$ & $\begin{array}{c}\quad 6.5 \times 10^{-16} \tau^{3 / 2} \\
\text { for } 1 \mathrm{~km} \text { orbital error and } \\
10 \mathrm{~s} \leq \tau \leq 300 \mathrm{~s}\end{array}$ & \\
\hline
\end{tabular}

\section{Simulation and Data Processing Software}

The simulation software is a Matlab package developed by the ACES collaboration (Meynadier et al. 2018). Its inputs are: orbitography of the ISS, terrestrial coordinates of a set of GS's and a model of the geo-potential in order to compute the relation between the proper times given 
by the ISS and GS's on the one hand, and coordinate time on the other. The total time-offlight is the sum of a geometric time-of-flight obtained by solving a two-point boundary value problem in GR, an ionospheric delay computed using the atmospheric electron density profile in a Chapman layer model together with a dipolar model for the earth's magnetic field, a tropospheric delay modeled using the Saastamoinen model, and a Shapiro delay. The pToF is then obtained from the total ToF and the coordinate to proper time transformations for the GSs and the ISS. These pToFs can then be used to compute the MWL's observables, which are based on local signal beat notes and counter measurements.

The data processing software is a Python package also developed by the ACES collaboration (Meynadier et al. 2018). The processing step that computes desynchronization from pToFs operates as shown in Fig. 3. As it is comparatively more straightforward once the necessary equations are derived, we shall not discuss here the pre-processing step that computes pToFs from the MWL raw data. Processing of the pToFs initially starts from MWL data in a non- $\Lambda$ configuration, i.e. the configuration that is realized in practice. As depicted in the figure, initial range estimates are obtained from orbitography data for the ISS and the positions of the GS's. These estimates are used together with atmospheric models and atmospheric data to produce tropospheric and ionospheric delays. An orange arrow indicates that, once computed, the atmospheric delays are used to refine the initial range estimates. By combining those refined range estimates with the pToFs, an estimate of desynchronization can be obtained and used, together with ToFs, to obtain the $\Lambda$ configuration. The pToF data is then re-computed in the $\Lambda$ configuration and produces the final desynchronization.

The performance of the data processing software is shown in Fig. $\mathbf{4}$ for a single pass of the ISS over a GS from a dataset generated by the simulation software. The figure demonstrates that the data processing software recovers the input of the simulation at the ps level and that the temporal Allan deviation complies with the ACES requirements shown in Fig. 1. 


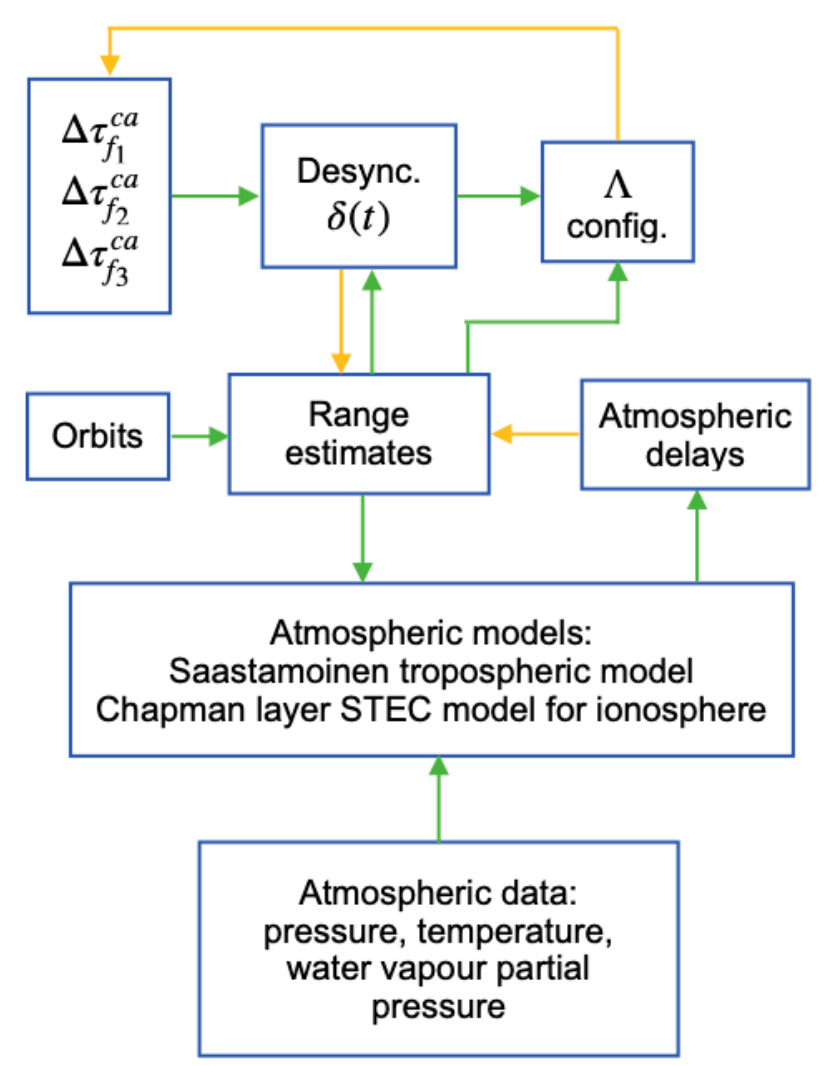

Fig. 3 ACES MWL data processing pipeline. The main output of the processing software is desynchronization. The pre-processing step, that computes the pToFs from the MWL raw data is not represented in this figure. Note that orange arrows highlight the iterative nature of some of the data processing steps. 

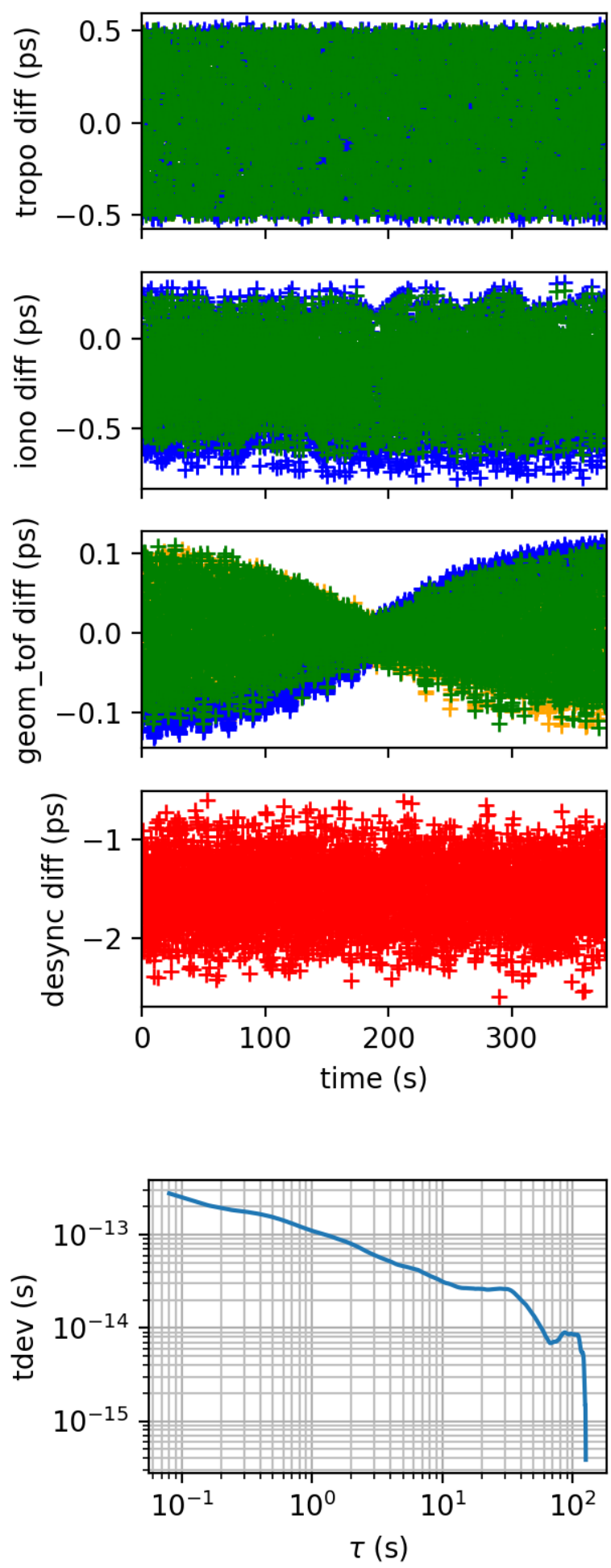

Fig. 4 Difference between simulation input and data processing output for some physical quantities, for a single ISS pass. The top 3 plots show time series of the tropospheric delay, the ionospheric delay and the geometric time of flight (ToF) for the uplink (blue) and the two downlink (green and yellow) signals. The last two plots show the desynchronization and its temporal Allan deviation (Time deviation). 


\section{Gravitational Redshift}

Albert Einstein's General Relativity (GR) establishes a link between gravitation and geometry by describing gravitation as a curvature of space-time. This geometrical description of the gravitational interaction relies on the Einstein Equivalence Principle (EEP) which postulates the equivalence of all inertial observers. To unify GR and the Standard Model (SM) of particle physics, small deviations from the EEP are expected. There are a variety of experiments seeking to detect those deviations. Three of the implications of the EEP can be tested experimentally (Will 2018):

- The universality of free fall, which states that the trajectory of a body is independent of its structure and composition (Touboul et al. 2017; Wagner et al. 2012),

- Local Lorentz invariance, which states that the outcome of any local non-gravitational experiment is independent of the velocity of the observer (norm and direction),

- Local position invariance, which states that the outcome of any local non-gravitational experiment is the same wherever it is performed in space-time.

The latter can be tested either by looking for variations of the fundamental constants (Uzan 2011; Will 2018) or with gravitational redshift tests.

As predicted by GR, a clock close to a massive body ticks slower than a clock far from it, thus creating a gravitational "redshift". For PHARAO and a ground clock, the clocks' relative frequency difference will be affected by two main effects, as seen in the introduction:

- The clocks' velocities in the geocentric non-rotating coordinate system lead to a second-order Doppler effect as predicted by Special Relativity (implying local Lorentz invariance).

- The clocks' positions in different gravitational potentials lead to a gravitational redshift (implying local position invariance).

While the latter will speed up PHARAO's rate compared to a ground clock, the former will slow it down. For a ground clock and PHARAO/SHM onboard the ISS (at an altitude of about 350 $\mathrm{km}$, and a velocity of about $8 \mathrm{~km} / \mathrm{s}$ ), the differential gravitational redshift is 10 times smaller and opposite in sign from the Doppler effect, so that the overall clock rate of PHARAO is slower 
than that of a static clock on the ground. As emphasized in previous sections, the scientific observable of the mission is clock desynchronization, which is the integral of the relative frequency difference. The effect of the gravitational redshift on desynchronization corresponds to a drift of about $3 \mu$ s per day as can be seen in the upper plot of Fig. $\mathbf{5}$. The orbit of the ISS above a ground clock induces a modulation of the range separating the GS and ISS clocks (shown in the red curve of the lower plot of Fig. 5). The corresponding oscillation of the relative frequency difference (the blue curve in the plot) is a consequence of the gravitational redshift as the ISS orbits around the earth. The slight eccentricity of the orbit as well as higher order terms in the earth's gravitational potential (e.g. earth flattening) give rise to the periodic features, whereas the offset (about $3.64 \times 10^{-11}$ ) corresponds to the leading $G M / r c^{2}$ term.

As explained in the introduction, to test local position invariance, the Einstein's gravitational redshift formula is rescaled by a factor $1+\alpha$ with $\alpha=0$ for GR (Will 2018; Blanchet and Wolf 2016). Gravitational redshift was first measured in the Pound-RebkaSnider experiment (Pound and Rebka 1959; Pound and Rebka 1960; Pound and Snider 1965). Since then, the most accurate tests of the gravitational redshift to date have been performed in:

- The Gravity Probe A experiment (Vessot and Levine 1979; Vessot et al. 1980; Vessot 1989): a hydrogen maser clock onboard a rocket was compared to ground hydrogen masers with a continuous two-way microwave link, reaching an uncertainty of about $1.4 \times 10^{-4}$ after a two hours flight.

- the GREAT experiment (Galileo gravitational Redshift test with Eccentric sATellites) (Delva et al. 2015; Delva et al. 2018; Herrmann et al. 2018): clocks onboard two eccentric Galileo satellites were compared to ground clocks reaching an uncertainty of $2.5 \times 10^{-5}$.

Assuming the measurement is limited only by systematic effects on PHARAO's frequency, the sensitivity of the gravitational redshift test would reach an uncertainty on $\alpha$ of about 2 to $3 \mathrm{ppm}$, given roughly by the absolute accuracy of PHARAO in relative frequency $\left(10^{-16}\right)$ divided by the size of the effect $\left(3.6 \times 10^{-11}\right)$. 

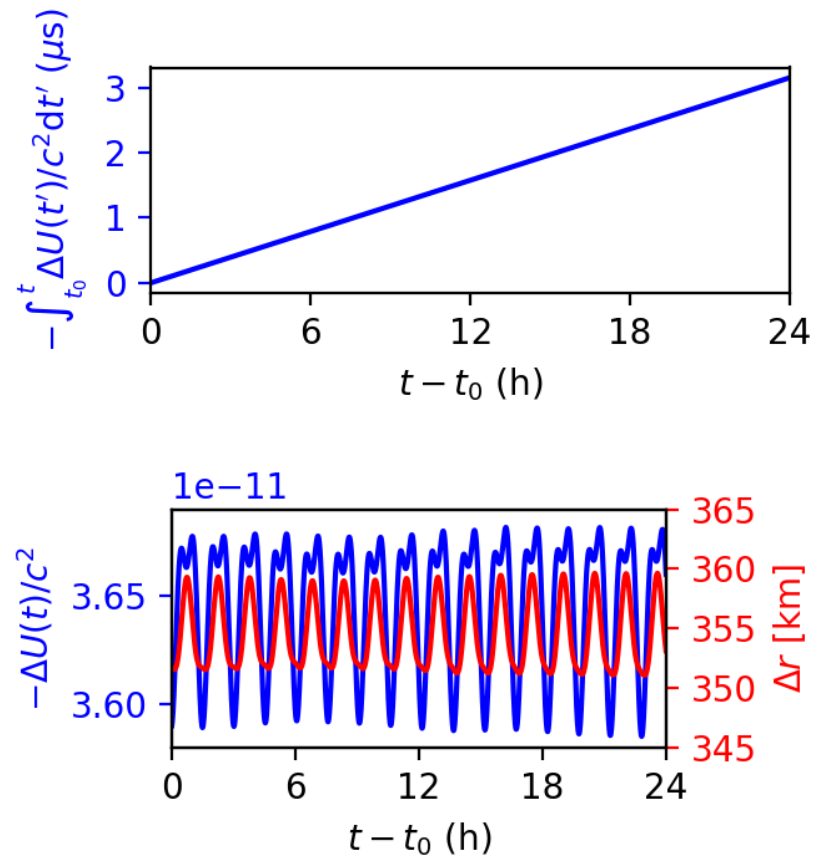

Fig. 5 Gravitational redshift model over one day for phase data (top) and for frequency (bottom) data, with on the right-hand axis the range between the ISS and the GS (Savalle et al. 2019).

\section{Scientific Data Analysis Software}

In addition to the data simulation software designed to reproduce realistic ACES observables and the data processing software designed to compute desynchronization data from those observables, both described in the section above, we have developed independent software that models desynchronization data and determines $\alpha$ from the data (Savalle et al. 2019):

- Desynchronization data is simulated from the GR model, including the correction brought by deviations from GR, using realistic ISS orbitography, a network of ground stations, the "Earth Gravitational Model" (Pavlis et al. 2008), mission noise specification and data gaps. Atmospheric effects and actual MWL observables, having been modeled and tested in the simulation and data processing software dedicated to the MWL are excluded from the simulation.

- In the data analysis software, the theoretical model is subtracted from the simulated data in order to compute the residuals and the model matrix linked to $\alpha$. 
- In order to estimate the value of the parameter $\alpha$, we use a Monte Carlo least squares method on a set of ACES simulations. Each realization includes the two dominant sources of noise in ACES: the MWL noise and the PHARAO/SHM clock noise. Both are specified by the mission requirements on their time and Allan deviations respectively (Table 2 and the associated discussion provides further details on the noise budget). We then fit the gravitational redshift model using an ordinary least squares method to obtain an estimate of $\alpha$. We repeat the fitting process a large number of times in order to obtain the probability distribution of the parameter $\alpha$ from which the standard deviation $\sigma_{\alpha}$ is computed. The quantity $\sigma_{\alpha}$ is the statistical uncertainty on $\alpha$ and it depends, for the durations of interest, which last 10 to 20 days, mainly on PHARAO's noise level and on measurement duration.

Both the simulation and its analysis were performed using real ISS orbitography data in batches of 12 days. Within these batches, the position of the ISS was interpolated to reach the ACES MWL sampling rate $(12.5 \mathrm{~Hz})$.

Phase and Frequency Data Analysis

In order to assess the performance level of the mission, we compared the uncertainty on $\alpha$ when using the phase data (i.e., the desynchronization data) and when using frequency data (i.e. using the relative frequency difference, given by the numerical derivative of the desynchronization data). For phase data and a 12-day simulation, the uncertainty on $\alpha$ is equal to $3 \times 10^{-6}$ (close to the target, $2 \times 10^{-6}$ ) but it falls short by 2 orders of magnitude in frequency (reaching only $5 \times 10^{-4}$ ). This difference can be explained as follows. While for phase data for which desynchronization is a linear drift, $\alpha$ is determined from a linear model for which the end points of the data set play a dominant role, for frequency data, $\alpha$ is determined from a constant offset, and its uncertainty depends mainly on the number of available data points and is thus strongly affected by the presence of data gaps. Given the limited visibility of the ISS by ground clocks, the number of points considered for the analysis in frequency is very limited. We, therefore, favor the analysis of desynchronization data.

Finally, our study demonstrates that the statistical uncertainty on $\alpha$ will reach $3 \times 10^{-6}$ after 12 days, in compliance with expectations. In addition, by combining a few 10 
to 20-day datasets, the results of Delva et al. (2018) in the GREAT experiment can be improved by an order of magnitude, assuming that systematic effects on PHARAO are indeed under control at the $1 \times 10^{-16}$ level.

\section{ISS Orbitography}

Because redshift scales as the inverse of the distance, achieving an uncertainty of about $10^{-6}$ on $\alpha$ requires a level of uncertainty of the ISS position of order $0.3 \mathrm{~m}$ for an average altitude of $350 \mathrm{~km}$. As already discussed, less stringent constraints, of order $10 \mathrm{~m}$, were obtained by Duchayne et al. (2009) using theoretical orbit error models. In this section, we carry out the same analysis with a real orbit of the ISS with a focus on the detection of the parameter $\alpha$. For this, two orbitography files obtained with two different methods are used. The difference in position vectors between the two files provides a realistic estimate of the ISS orbitography error. Its typical standard deviation is about $30 \mathrm{~cm}$, see Fig. 6.

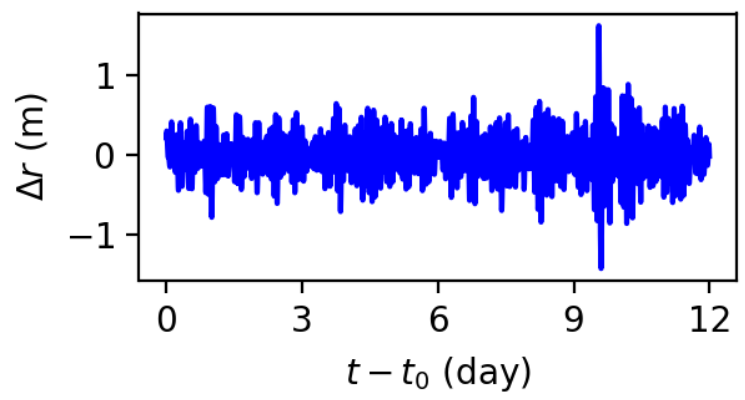

Fig. 6 ISS orbital error over 12 days starting at a reference time $t_{0}$ (Savalle et al. 2019).

We simulate 12-day data using the precise orbitography files and perform the analysis using orbitography files degraded such that the standard deviation of the orbital error is increased from $30 \mathrm{~cm}$ to $300 \mathrm{~km}$. We distinguish between simulations made with MWL and clock noise (empty circles in Fig. 7) and without noise (half-full circle).

The target uncertainty on $1+\alpha$ is plotted as a horizontal line. As can be seen in the figure, the measured mean value of $\alpha$ is a linearly increasing function of the orbital error $\left|\sigma_{r}\right|$ and a significant detection of $\alpha$ (i.e. above $3 \times 10^{-6}$ ) can be distinguished from a bias caused 
by the orbital error as long as $\left|\sigma_{r}\right| \leq 300 \mathrm{~m}$. As the present orbitography precision is of order of $30 \mathrm{~cm}$, the redshift test will easily be achieved without any systematic effect caused by a poor ISS orbitography. Note that this is a significantly less stringent requirement than the one obtained in Duchayne et al. (2009) in which the focus was for the Allan deviation of desynchronization to remain below PHARAO/SHM's performance for all integration times.

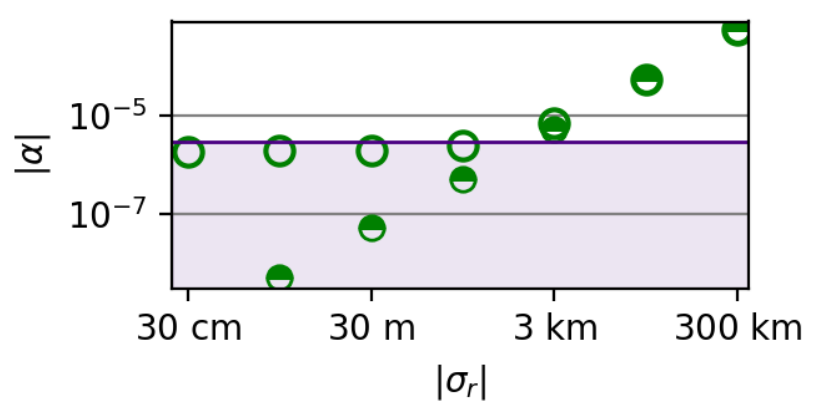

Fig. 7 Absolute values of $\alpha$ estimated with degraded orbitography with noise (empty circle) or without noise (half-full circles). The horizontal violet line is the statistical uncertainty on $\alpha$ under which the shadowed region defines the non-significance zone of $\alpha$ (Savalle et al. 2019).

\section{Ground Clock Network}

The mission will benefit from a network of up to $8 \mathrm{MWL}$ ground stations (GS) connected to the best available clocks in several metrology laboratories. To assess the benefit of this network, we compared the result obtained from each GS, and from a combined fit for all GS's. No significant statistical improvement on the determination of $\alpha$ is obtained with the phase data using the full network instead of a single station (Savalle et al. 2019). This is a consequence of the phase model, which depends mainly on the time elapsed between the beginning and the end of the mission and not on the amount of data in between. The addition of stations does not increase the mission duration but simply reduces the impact of data gaps. Therefore, no improvement is expected in the evaluation of $\alpha$ with the phase data if more than one station is used to perform the test. This being said, the use of the entire network is necessary to ensure robustness and to evaluate all systematic effects. 


\section{Conclusion}

The ACES mission uses a novel high-performance clock ensemble in space, aboard the ISS, PHARAO/SHM, and a network of high-performance ground clocks together with an MWL and a laser link (ELT, not discussed here) to enable state-of-the-art clock comparisons and tests of fundamental physics, such as the measurement of the gravitational redshift and possible deviations from GR.

From the experimental standpoint, one particularity of this mission, which should be noted is that the MWL is an integral part of the measurement process, as discussed in the introduction and illustrated in the right-hand plot of Fig. 1. For this reason, in the first part of this review, we focused our attention on the MWL and in particular, on the computation of desynchronization from basic MWL observables. We provided an overview of the methodology used to compute desynchronization from pToFs, as well as the associated simulation and data processing software developed and showed that the data processing code developed recovers the time delays and desynchronization generated by the simulation code at the ps level, with a temporal Allan deviation complying with requirements.

In the second part of this review, we then discussed one of the main scientific goals of the ACES mission, namely the gravitational redshift test. Under the assumption that PHARAO's systematic uncertainty is of order $10^{-16}$ in fractional frequency, the ACES space mission will reach a performance on the measurement of the gravitational redshift, and possible deviations away from GR of about 2 to $3 \times 10^{-6}$. By developing dedicated simulation and data analysis codes, we determined that this objective will be reached in a realistic scenario that includes numerous data gaps due to the ISS orbit and the noise due to PHARAO and the MWL. We showed that an inaccuracy in the orbitography of the ISS will have no impact on the precision with which the measurement of the gravitational redshift will be performed as long as it is less than $300 \mathrm{~m}$. We also note that the use of a single station does not affect the statistical evaluation of the GR violation parameter $\alpha$ but will be limiting for the estimation of systematic effects. 


\section{Acknowledgments}

We thank Christophe Salomon for his continued investment as PI of the ACES mission, Luigi Cacciapuoti for his dedication as the ACES project scientist at ESA, and Philippe Laurent for his long standing work as PI of the PHARAO cold atom clock.

\section{Data Availability}

All simulated data and the results of the data analysis can be made available upon request to the corresponding author.

\section{References}

Bassiri S, Hajj G (1993) Higher-order ionospheric effects on the global positioning system observables and means of modeling them. Manuscripta Geodaetica 181:280

Blanchet L, Wolf P (2016) Analysis of Sun/Moon gravitational redshift tests with the STEQUEST space mission. Class. Quant. Grav. 33:035012

Cacciapuoti L, et al. (2020) Testing gravity with cold-atom clocks in space. Europhys Lett. D. 74:164.

Delva P et al. (2018). Gravitational Redshift Test Using Eccentric Galileo Satellites. Phys. Rev. Lett. 121:231101

Delva P, Hees A, Bertone S, Richard E, Wolf P (2015) Test of the gravitational redshift with stable clocks in eccentric orbits: application to Galileo satellites 5 and 6 . Class. Quant. Grav. 32:232003

Delva P, Meynadier F, Wolf P, Le Poncin Lafitte C, Laurent P. (2015) Time and frequency transfer with a microwave link in the ACES/PHARAO mission. In: 28th General Assembly of the International Astronomical Union, Highlights Astron. 16:211

Duchayne L. Mercier F, Wolf P (2009) Orbit determination for next generation space clocks. A\&A 504:653-661

Herrmann S, et al. (2018) Test of the Gravitational Redshift with Galileo Satellites in an Eccentric Orbit. Phys. Rev. Lett. 121:231102 
Hobiger T, Piester D, Baron P (2013) A correction model of dispersive troposhere delays for the ACES microwave link. Radio Science 48(2):131

Laurent, P, et al. (2006) Design of the cold atom PHARAO space clock and initial test results. Appl. Phys. B 84:683

Meynadier F, Delva P, le Poncin Lafitte C, Guerlin C, Wolf, P (2018) Atomic Clock Ensemble in Space (ACES) data analysis. Class. Quant. Grav. 3:035018

Pavlis N, Holmes S, Kenyon S, Factor J (2008) The development and evaluation of the Earth Gravitational Model 2008. Journal of Geophysical Research: Solid Earth 117:B4

Pound R, Rebka G (1959) Gravitational redshift in nuclear resonance. Phy. Rev. Lett. 3:439

Pound R, Rebka G (1960) Apparent weight of photons. Phys. Rev. Lett. 4:337

Pound R, Snider J (1965) Effect of gravity on Gamma radiation. Phys. Rev. B 788

Saastamoinen, J (1973) In: Contributions to the theory of atmospheric refraction, Bulletin Geodesique 47:13

Savalle E, Guerlin C, Delva P, Meynadier F, Le Poncin Lafitte C, Wolf P (2019) Gravitational redshift test with the future ACES mission. Class. Quant Grav. 24:245004

Touboul P, et al. (2017). MICROSCOPE. Phys. Rev. Lett. 119:231101

Uzan J (2011) Varying Constants, Gravitation and Cosmology. Living Reviews in Relativity $14: 2$

Vessot R, et al. (1980) Test of Relativistic Gravitation with a Space-Borne Hydrogen Maser. Phys. Rev. Lett. 45:2081

Vessot R, Levine M (1979) A test of the equivalence principle using a space-borne clock. General Relativity and Gravitation 10:181

Vessot, R (1989) Clocks and spaceborne tests of relativistic gravitation. Advances in Space Research 9:21

Wagner T, Schlamminger S, Gundlach J, Adelberger E (2012) Torsion-balance tests of the weak equivalence principle. Class. Quant. Grav. 29:184002

Will C. (2018) Theory and Experiment in Gravitational Physics. Cambridge University Press 